\title{
A Practical Guide for Managing Interdisciplinary Teams: Lessons Learned from Coupled Natural and Human Systems Research
}

\author{
V. Reilly Henson ${ }^{1}{ }^{(D)}$, Kelly M. Cobourn ${ }^{2, *} \mathbb{( D}$, Kathleen C. Weathers ${ }^{3}$, Cayelan C. Carey ${ }^{4}(\mathbb{D}$, \\ Kaitlin J. Farrel1 ${ }^{5}$, Jennifer L. Klug ${ }^{6}$, Michael G. Sorice ${ }^{2}(D)$, Nicole K. Ward ${ }^{4}\left(\mathbb{D}\right.$ and Weizhe Weng ${ }^{7}$ \\ 1 Hume Center for National Security and Technology, Virginia Tech, Blacksburg, VA 24061, USA; \\ vrhenson@vt.edu \\ 2 Department of Forest Resources and Environmental Conservation, Virginia Tech, Blacksburg, VA 24061, \\ USA; msorice@vt.edu \\ 3 Cary Institute of Ecosystem Studies, Millbrook, NY 12545, USA; weathersk@caryinstitute.org \\ 4 Department of Biology, Virginia Tech, VA 24061, Blacksburg, VA 12545, USA; cayelan@vt.edu (C.C.C.); \\ nkward@vt.edu (N.K.W.) \\ 5 Odum School of Ecology, University of Georgia, Athens, GA 30602, USA; kfarrell@vt.edu \\ 6 Department of Biology, Fairfield University, Fairfield, CT 06824, USA; jklug@fairfield.edu \\ 7 School of Business, SUNY Geneseo, Geneseo, NY 14454, USA; wweng@geneseo.edu \\ * Correspondence: kellyc13@vt.edu
}

Received: 12 May 2020; Accepted: 6 July 2020; Published: 9 July 2020

\begin{abstract}
Interdisciplinary team science is essential to address complex socio-environmental questions, but it also presents unique challenges. The scientific literature identifies best practices for high-level processes in team science, e.g., leadership and team building, but provides less guidance about practical, day-to-day strategies to support teamwork, e.g., translating jargon across disciplines, sharing and transforming data, and coordinating diverse and geographically distributed researchers. This article offers a case study of an interdisciplinary socio-environmental research project to derive insight to support team science implementation. We evaluate the project's inner workings using a framework derived from the growing body of literature for team science best practices, and derive insights into how best to apply team science principles to interdisciplinary research. We find that two of the most useful areas for proactive planning and coordinated leadership are data management and co-authorship. By providing guidance for project implementation focused on these areas, we contribute a pragmatic, detail-oriented perspective on team science in an effort to support similar projects.
\end{abstract}

Keywords: authorship; collaboration; data management; interdisciplinary research; project management; teamwork; team science

\section{Introduction}

Science increasingly relies upon team-based, interdisciplinary research (Van Noorden 2015; Wuchty et al. 2007) to address complex issues in socio-environmental systems (Palmer et al. 2016), which are "tightly linked social and biophysical subsystems that mutually influence one another" (SESYNC 2012). Climate change, species conservation, and natural resource management are just a few examples of complex socio-environmental issues that bring together disciplines such as biology, chemistry, physics, and psychology, among others (Liu et al. 2007). 
Integrating disciplines requires overcoming significant challenges. Each discipline has distinctive jargon, methods, cultures, training, and philosophies, often leading to perceived incompatibilities with other disciplines and ways of knowing, and practical challenges in achieving shared goals (Eigenbrode et al. 2007). While these obstacles can be overcome with careful communication and team building (Bennett and Gadlin 2012), they are compounded by the additional logistical challenges of teamwork, such as time management, progress tracking, and information sharing. There is a movement towards sharing the lessons learned from projects that successfully achieve their objectives, in order to develop best practices for similar efforts (e.g., Carey et al. 2019; Stokols et al. 2008). For example, team diversity (e.g., career stage, discipline, gender) and teamwork exercises can help to build interpersonal skills and improve team productivity (Cheruvelil et al. 2014). Similarly, leadership, communication, and goal-defining have been identified as essential in successful interdisciplinary research (Alberti et al. 2011; Lanier et al. 2018).

We contribute to this growing effort to understand the challenges of interdisciplinary integration by sharing a case study of our diverse interdisciplinary team's coupled natural and human systems (CNHS) project. This project required extensive interdisciplinary integration among members of a sizeable, diverse, and geographically distributed team. We focus our discussion on two particular processes that we found to be essential to the successful creation of multidisciplinary knowledge: data management and co-authorship. We define data management as the process of ensuring the accessibility and reliability of data files for users within and across disciplinary sub-teams. Not only is this process instrumental to successful knowledge creation within our team; it also has implications for the impact and replicability of research more broadly (Wilkinson et al. 2016). Co-authorship refers to the process of collaboratively creating a product, such as a journal article, poster, presentation, or dataset. As knowledge is created through a project such as ours, co-authorship practices require that credit be assigned appropriately and accurately.

Here, we share advice and experiences in an effort to aid the efficiency of future research teams. When researchers are forthcoming about which project management strategies worked for their teams and which did not, it provides other researchers with the opportunity to make more informed decisions going into their own interdisciplinary team science endeavors (Frassl et al. 2018; Oliver et al. 2018). While the lessons we learned are likely to be particularly relevant to other CNHS researchers, they have value for interdisciplinary science teams working in other contexts.

\section{CNHS Case Study Background}

Our project (henceforth referred to as "CNH Lakes") studied lake water quality as part of a five-year project funded by the U.S. National Science Foundation (NSF). Our objective was to examine the connections between lake water quality, surrounding land use, and local communities by linking multiple disciplinary analyses and models. To accomplish this, we drew upon the disciplines of agronomy, hydrology, ecology, economics, and sociology. Researchers from each discipline modeled specific components of human-lake systems (e.g., how nutrient inputs affect water quality and how water quality affects the monetary value of lakefront properties), then disciplinary models and analyses were coupled to simulate a feedback loop between lakes and their surrounding communities. Coupling the models involved addressing differences between disciplinary approaches along multiple dimensions, including bridging quantitative and qualitative approaches, reconciling the spatial and temporal scales of process and models, and bridging between mechanistic (process-based) and statistical methodologies (Cobourn et al. 2018).

Our project focused on two lakes in the United States: Lake Mendota in Wisconsin, and Lake Sunapee in New Hampshire. Using our coupled modeling system for these lakes, we explored how changes in one part of the human-lake system affected others. We also scaled up this understanding using statistical methods to determine the extent to which conclusions drawn from these study lakes could be extrapolated to thousands of similar lakes in the northeastern and midwestern United States (Soranno et al. 2017). 
Our team included over 20 members spanning a variety of career stages, institutions, and disciplinary backgrounds. Over the duration of the project, the team fluctuated in size and disciplinary composition as researchers joined, left, and progressed through career stages. This diversity lent creativity and innovation to the team process (Cheruvelil et al. 2014), but required additional coordination and management to avoid logistical and communication pitfalls, and to ensure that the workflow went smoothly. This feature is likely to be consistent among CNHS research efforts, and in many other types of interdisciplinary research projects. Within our team, eight sub-teams of researchers focused on specific disciplinary models or analyses. Sub-teams ranged from relatively small (e.g., one professor and one graduate student working on a specific model for each study lake) to somewhat large relative to our total team size (e.g., four professors, a postdoc, and several graduate students working on the same model, but specializing in different lakes or analyses). Some researchers were members of multiple sub-teams. All researchers in the team collaborated on simulating the feedback loop between human and natural systems.

\section{Challenges and Lessons Learned from the Case Study}

To evaluate our case study, we build on the conceptual framework presented in a National Academy of Sciences (NAS) report, which is one of the most comprehensive reviews of best practices for team science to date (National Research Council 2015). The report synthesized findings from the literature on the "science of team science" and provided a framework for understanding the challenges that science teams face. The NAS framework categorizes challenges according to the seven dimensions of team science from which they are most likely to arise: (1) high diversity of membership; (2) deep knowledge integration; (3) large team size; (4) goal misalignment with other teams; (5) permeable boundaries; (6) geographic dispersion; and (7) high task interdependence. Our team dealt with a variety of challenges that closely matched these seven dimensions.

We use the NAS framework to organize the discussion of our experiences in the remainder of this section. These experiences were gathered systematically from this article's set of co-authors, as well as through informal conversations with the broader project team. Table 1 summarizes our team's successes and challenges in the areas of data management and co-authorship along each dimension identified in the NAS report. Table 1 also presents recommended practices based on lessons learned from our case study.

Table 1. Summary of challenges and lessons learned in each dimension of team science ${ }^{1}$.

\begin{tabular}{|c|c|c|c|c|}
\hline Dimension & Definition & $\begin{array}{l}\text { Application to Data } \\
\text { Management }\end{array}$ & Application to Co-Authorship & Recommended Practices \\
\hline $\begin{array}{l}\text { High diversity } \\
\text { of membership }\end{array}$ & $\begin{array}{l}\text { Disciplinary diversity, as } \\
\text { well as diversity in culture, } \\
\text { age, gender, religion, } \\
\text { ethnicity, career stage, and } \\
\text { more }\end{array}$ & $\begin{array}{ll}\text { - } & \text { Diverse data storage and } \\
\text { sharing needs }\end{array}$ & $\begin{array}{ll}- & \text { Distinct disciplinary norms } \\
- & \text { Reconciling norms for } \\
& \text { interdisciplinary manuscripts }\end{array}$ & $\begin{array}{l}\text { Early project workshops to } \\
\text { share data management needs } \\
\text { and strategies } \\
\text { Plan for co-author } \\
\text { manuscript contributions } \\
\text { - Living authorship policy }\end{array}$ \\
\hline
\end{tabular}


Table 1. Cont.

\begin{tabular}{|c|c|c|c|c|}
\hline Dimension & Definition & $\begin{array}{c}\text { Application to Data } \\
\text { Management }\end{array}$ & Application to Co-Authorship & Recommended Practices \\
\hline $\begin{array}{l}\text { Goal } \\
\text { misalignment }\end{array}$ & $\begin{array}{l}\text { Individuals, sub-teams, } \\
\text { groups, and institutions } \\
\text { with a variety of goals and } \\
\text { incentives that may be in } \\
\text { conflict with one another }\end{array}$ & $\begin{array}{l}\text { - Work-related obligations in } \\
\text { addition to CNH Lakes } \\
\text { Occasionally challenging to } \\
\text { find time for CNH Lakes } \\
\text { data management tasks }\end{array}$ & $\begin{array}{l}\text { - } \begin{array}{l}\text { Different publication } \\
\text { priorities for different }\end{array} \\
\text { career stages } \\
\text { - Conflicting incentives for } \\
\text { co-authors on a } \\
\text { single publication }\end{array}$ & $\begin{array}{l}\text { - Adapt distribution of tasks to } \\
\text { individuals who can realistically } \\
\text { work on them } \\
\text { Encourage team members to } \\
\text { share concerns related to career } \\
\text { stage priorities }\end{array}$ \\
\hline $\begin{array}{l}\text { Permeable } \\
\text { boundaries }\end{array}$ & $\begin{array}{l}\text { The joining and/or leaving of } \\
\text { some team members } \\
\text { throughout a project's } \\
\text { lifetime }\end{array}$ & $\begin{array}{l}\text { Loss of dataset knowledge } \\
\text { when members leave } \\
\text { the team }\end{array}$ & $\begin{array}{l}\text { Determination of whether } \\
\text { new members should } \\
\text { become co-authors on } \\
\text { existing manuscripts }\end{array}$ & $\begin{array}{l}\text { - Carefully document all work } \\
\text { and data } \\
\text { - Update research objectives as } \\
\text { team evolves } \\
\text { Establish protocol for } \\
\text { onboarding new team members }\end{array}$ \\
\hline $\begin{array}{l}\text { Geographic } \\
\text { dispersion }\end{array}$ & $\begin{array}{l}\text { Team members who live and } \\
\text { work in different locations, } \\
\text { and at different institutions }\end{array}$ & $\begin{array}{l}\text { Complex data management } \\
\text { issues not easily resolvable } \\
\text { via remote communication }\end{array}$ & $\begin{array}{l}\text { Varying efficiency of online } \\
\text { tools for different } \\
\text { writing stages }\end{array}$ & $\begin{array}{l}\text { Create opportunities for } \\
\text { in-person meetings, } \\
\text { where possible } \\
\text { Switch between writing tools as } \\
\text { the need arises }\end{array}$ \\
\hline $\begin{array}{l}\text { High task } \\
\text { interdependence }\end{array}$ & $\begin{array}{l}\text { Team members depending } \\
\text { on each other to complete } \\
\text { tasks in order to move the } \\
\text { project forward }\end{array}$ & $\begin{array}{l}\text { - Model couplings with } \\
\text { many interdependent tasks } \\
\text { - Careful coordination of } \\
\text { timelines for sharing data }\end{array}$ & $\begin{array}{l}\text { Represent perspectives of } \\
\text { all co-authors while } \\
\text { maintaining cohesion } \\
\text { and progress }\end{array}$ & $\begin{array}{l}\text { - Have all co-authors contribute, } \\
\text { but having one or two leaders } \\
\text { who push the process forward } \\
\text { - Sub-teams work on } \\
\text { discipline-specific publications } \\
\text { while waiting for outputs from } \\
\text { other model(s) }\end{array}$ \\
\hline
\end{tabular}

${ }^{1}$ National Research Council (2015).

\subsection{Dimension 1: High Diversity of Membership}

Diversity on a science team is valuable, as it can provide a wide base of expertise, enhance creative problem solving, and bring multiple perspectives into conversation (Cheruvelil et al. 2014; Lee et al. 2015; Uriarte et al. 2007). This is true of diversity along many axes, including discipline, culture, age, race, gender, religion, and ethnicity. However, disciplinary diversity in particular has the potential to create communication barriers between team members (Eigenbrode et al. 2007). For an interdisciplinary project such as ours, breaking down these barriers between disciplines can constitute a significant expenditure of time and effort, but yields major benefits in terms of overall productivity and integration. Figure 1 illustrates the diversity of our team across disciplines at two points during the project's duration.

\begin{tabular}{|c|c|c|c|}
\hline $\begin{array}{l}\text { Disciplinary } \\
\text { background }\end{array}$ & Team in Year 1 & Team in Year 3 & \\
\hline Agronomy & ( & & \\
\hline Hydrology & & & $\mathrm{PhD}$ student \\
\hline Ecology & & & Technician/other \\
\hline Economics & & & Assistant professor \\
\hline Sociology & & & Full professor \\
\hline $\begin{array}{l}\text { Other (data science, } \\
\text { management, etc.) }\end{array}$ & & & \\
\hline
\end{tabular}

Figure 1. Distribution of CNH Lakes team members' backgrounds and career stages. 


\subsubsection{Diversity as a Challenge for Data Management: Establishing Data-Sharing Conventions}

Broadly speaking, no method is universally adopted for characterizing and managing data (Rüegg et al. 2014). Different disciplines—and even different groups within a discipline — tend to have their own preferences and conventions. This can include different file naming practices, controlled vocabulary, platforms for hosting files, and ways of formatting documents and tables. In an interdisciplinary project where data are passed between sub-teams for modeling and analyses, this means either that the entire project team must adopt a single standard for storing and sharing data, or that files must be adapted and moved at each transition between models.

In the $\mathrm{CNH}$ Lakes project, there was substantial variation among disciplinary sub-teams in terms of file sizes (sometimes exceeding hundreds of gigabytes), preferences for data storage platforms, and human subject confidentiality requirements. Therefore, when sharing working versions of our files, we often defaulted to using whatever platform could serve as a "common denominator" between sub-teams. Generally, this meant using a commercial web platform that was accessible, familiar, and secure for all researchers involved in a particular model coupling. Exchanging data from human subjects between team members required adhering to Institutional Review Board (IRB) protocol by ensuring that shared and archived data were anonymized or aggregated (e.g., with summary statistics). Challenges also arose with the use of proprietary data obtained from third-party vendors, which required that we shared and archived only summary statistics and results, rather than the raw observations used in the analysis.

By maintaining the flexibility for sub-teams to choose their preferred file sharing platforms, we ensured that their distinct needs at each model coupling were met to the extent possible. However, some of these platforms lacked sufficient features for tracking which version of a file was most up to date. For this reason, toward the end of the project we began encouraging team members to use a platform with greater versioning control capacity (see Section 3.3.1). This was a challenge because not all team members were familiar with the use of these types of platforms.

We undertook a separate effort to establish a permanent data archive, which was intended to provide a record of the data used in the final version of our models, to facilitate the replicability of our project. This archive did not need to be as flexible as our storage strategy for working files, because it did not need to host working versions of files or to be frequently updated. For this reason, differences among disciplines were not as relevant.

Discrepancies in data management techniques and associated pitfalls of the "common denominator" approach could potentially be alleviated by including the whole team in a brainstorming session at the start of the project to create a sense of ownership over the ultimate solution for file management. This encourages buy-in and reduces resistance to learning to use new platforms. Subsequently, holding a series of data management training workshops could ensure that all team members have a shared language and goal when it comes to project-wide practices. With enough research, team leadership may even be able to determine a single data-sharing platform and define a set of best practices that could be used efficiently across disciplines and institutions.

\subsubsection{Diversity as a Challenge for Co-Authorship: Reconciling Disciplinary Norms}

Through experience, we found that many disciplines have their own distinct cultures and expectations (sometimes unwritten, but understood) surrounding authorship. While these practices can help to provide structure and maintain rigor among publications within a discipline, they also can create challenges when multiple disciplines collaborate on a single manuscript. We observed this in the form of widely differing norms and conventions primarily about the number of co-authors on a given paper, and to a lesser extent the order in which authors should be listed. For example, articles in economics and the social sciences tend to have a relatively small set of co-authors (on the order of one to four), whereas it is increasingly common in the field of ecology to have many co-authors (sometimes in the dozens to even hundreds of authors). 
These differences in expectations and practices are especially apparent when graduate students are involved as co-authors. Some disciplines are more accepting of having co-authors on student dissertation chapters than others. Furthermore, we have found that different disciplines tend to support different levels of graduate student integration into the full project team. In some cases, a graduate student becomes fully immersed in the project as a whole, whereas other times a graduate student is brought on to complete a specific task and has limited engagement with larger project-wide tasks and analyses. Because of this range of involvement, graduate student participation in collaborative manuscripts may also be inconsistent across disciplines.

Ongoing, open conversations about authorship helped our team to address potential sources of authorship conflict. We developed a co-authorship policy early in the project (Appendix A), but recognized that our understanding of the differences in authorship norms between disciplines would evolve throughout our work. Therefore, we created a mechanism to revisit the policy quarterly. Through ongoing discussions, these differences gradually became clearer, and conversations about co-authorship became progressively easier as we developed a mutual understanding of each other's expectations.

As part of the authorship policy, our standard practice was to distribute a formalized memo to the entire team whenever a project-related manuscript was conceptualized (Appendix A). This provided a chance for all team members to discuss the possibility of becoming a co-author. Importantly, these authorship memos also served to communicate details about who should consider being a co-author. For instance, when some of our team members wrote a manuscript for a journal that limited the number of authors that can be listed, we included this information in the authorship memo, so that team members would understand these restrictions upfront. These memos were also opportunities to clarify when a manuscript was part of a thesis or dissertation chapter, which could influence co-authorship by restricting the number of authors, or the order in which authors would be listed.

We suggest that establishing a policy that encourages discussion at the inception of each manuscript about how each co-author and discipline will contribute and be given credit for their work reduces disagreements later on in the writing process and avoids the potential for unintended marginalization of collaborators (Frassl et al. 2018; Oliver et al. 2018). For example, author contribution statements included in manuscripts help to clarify what intellectual contributions were made by whom, both for the team and for readers (Goring et al. 2014).

\subsection{Dimension 2: Deep Knowledge Integration}

Interdisciplinary teams strive to integrate multiple types of data and methods to broaden perspectives and produce new, synthetic knowledge that could not be achieved by one discipline alone. The process of integration often requires problem solving, innovation, and close collaboration.

\subsubsection{Integration as a Challenge for Data Management: Coordinating Analyses across Scales}

A major challenge for our project was linking analyses across spatial and temporal scales. For instance, our agronomic model simulated crop growth at a sub-daily time scale. However, this model was coupled with an economic decision-making model that produced outputs at the seasonal (e.g., sub-annual) timescale. To integrate these disciplinary models, we had to aggregate simulated crop yield to an annual value in order to apply yearly economic decision-making predictions back to the agronomic model. This was an innovative approach that we were able to implement due to careful communication and planning, but took multiple years to develop.

Furthermore, when different models or analyses use distinct types of data, researchers must arrange and manage these data differently. For example, while most of our models used quantitative data, our analysis of volunteer environmental associations exclusively focused on qualitative data, which do not mirror the type of numerical modeling used in other parts of the project. In this case, the knowledge gained through qualitative analysis was used to bound scenarios in the real world, based on deep insight into the capacity and performance of volunteer groups. This allowed the human subjects' data to be synthesized in a way that added behavioral parameters into the integrated models. 
We were only able to achieve this integration through close collaboration between team members, with multiple in-depth meetings and brainstorming sessions, all the while ensuring that all parties maintained openness toward multiple disciplinary ways of knowing.

\subsubsection{Integration as a Challenge for Co-Authorship: Overcoming Communication Barriers}

Although an interdisciplinary project produces some discipline-specific publications, its most significant and innovative outputs are likely to involve deep knowledge integration among disciplines. Generally speaking, such publications are difficult to produce, both conceptually and practically. For example, during the process of developing a literature review paper that incorporated ecology, economics, and sociology (Ward et al. 2019), members of our team experienced challenges in integrating their knowledge of specific terminologies. In particular, it became clear while trying to distinguish between economic and socio-cultural processes that terms such as "preference" and "value" have different meanings in the economic and sociology literatures (Jones et al. 2016; Pascual et al. 2017). Significant effort was needed to establish a shared understanding of these concepts and integrate disciplinary perspectives. Even after much discussion, some co-authors "agreed to disagree". At times, the most expedient option may be for co-authors to acknowledge different disciplinary definitions of a shared term, and agree to use the definition established in the body of literature where the specific manuscript will be published, even if that definition is not how the term is used in one's own discipline. Even so, this process of deliberation and cross-disciplinary literature exploration resulted in a greater depth of integration within our project.

We found it helpful for co-authors from different disciplines to minimize the use of disciplinespecific jargon or language that could confuse collaborators, so that they could reduce the amount of time spent defining and disambiguating terms. This was generally accomplished indirectly, by setting an expectation at the outset that the target audience would be interdisciplinary, which encouraged co-authors to write in a way that would be accessible to readers in other disciplines. We also found it helpful for co-authors to have detailed discussions about terminology at the beginning of the project, and to establish a culture where team members were encouraged to ask each other for clarification when needed. For example, we found that different disciplines had varying definitions for certain commonly used words, such as "scenario". Economists tended to think of scenarios as the probable effects of certain variables, whereas ecologists tended to think of scenarios as possible situations or changes in the future. During our first in-person project workshop, we held a session to address the possible definitions of words such as these and to understand potential disciplinary differences.

\subsection{Dimension 3: Large Team Size}

Team science can be defined as any research conducted by two or more people working together (National Research Council 2015), though there is a distinction between small teams (two to ten individuals) and larger groups (more than ten individuals). The CNH Lakes team was a larger group, with roughly 20 team members at any given point (Figure 1). Having a team of this size can allow a project to include both expertise from different disciplines and multiple perspectives within individual disciplines (Lee et al. 2015). Broadly speaking, though, larger groups of researchers tend to require greater coordination in order to complete and integrate their tasks than smaller teams (Schimel and Keller 2015).

Over the course of CNH Lakes, various individuals took on project management tasks. Lead principal investigators (PIs), and then a postdoc, initially held the responsibilities of tracking progress, monitoring the budget, facilitating meetings, and planning events. At the end of the project's second year, a dedicated project manager position was created, allowing one person to take on these tasks, freeing up considerable time for the researchers. Hiring a project manager may not be viable for all research efforts, but clearly defining and distributing project management responsibilities is likely to help the research run more smoothly. 


\subsubsection{Size as a Challenge for Data Management: Practicing Versioning Control}

The most salient data management challenge that arose from the size of our team was maintaining versioning control, which refers to the process of tracking changes to a file over time to avoid ambiguity over which file is most up to date. Because we often had multiple people working together to analyze the same dataset, a single file would be revised several times by different individuals. For example, the lake water quality model used by the ecology sub-team required the fine-tuning of hundreds of lake-specific parameters during model calibration. Because of the model's structure, the parameter file always had to have the same name (e.g., "glm2.nml"; Hipsey et al. 2019), so team members could not easily distinguish file versions based on file names. Additionally, some files (such as long-term meteorological driver data) were so large that they could not easily be stored on some platforms, limiting the team's ability to track version history. These types of challenges generated confusion among sub-teams as to which file was the most current version, or which file was intended for use in a particular analysis.

Furthermore, different people often worked with similar data, but aggregated the data over different spatial or temporal scales, which added another dimension to the task of keeping files organized. For example, one of the economics sub-teams needed to aggregate lake water quality data collected each minute into an annual mean, whereas the ecologists needed to aggregate the data to the hourly scale, resulting in multiple files of the same base data but at different temporal scales.

According to the experience of several of our team members, versioning control is an ongoing issue for many interdisciplinary projects. A larger team size only tends to magnify this challenge. Our team attempted to address this issue by collaboratively creating a document that listed a single contact person for each type of dataset used in the project. Our goal was to streamline the process of communicating about data by placing one person in charge of monitoring and organizing version history. However, it remains unclear whether this had any detectable benefit to the team, because most sub-teams had already established their own practices for dealing with and communicating about data, and those did not appear to change substantially over the course of the project. One suggestion for improving this strategy would be to establish a list of dataset contacts at the beginning of a project, as opposed to after the project was already underway.

\subsubsection{Size as a Challenge for Co-Authorship: Keeping Momentum in Manuscript Development}

When developing a manuscript with a large number of co-authors, an important challenge is managing the workflow. For example, delays in writing by a single co-author can slow down the writing process for the entire group. Bottlenecks in our group were particularly problematic toward the end of manuscript development, when each co-author was asked to go through the document in detail and offer final revisions. We found that authorship memos provided useful opportunities to establish leadership, lay out goals and deadlines, and clearly define the roles of each contributor, so that delays were minimized, though rarely eliminated. Additionally, during final manuscript editing before submission, lead authors were encouraged to be clear about deadlines for incorporating edits and co-authors were responsible for communicating with the lead author if the deadline was not going to work with their schedule.

\subsection{Dimension 4: Goal Misalignment}

Individuals, sub-teams, research groups, and institutions are driven by different goals and incentives, which can at times conflict with one another. Our team did not experience a great deal of goal misalignment, which we attribute largely to our early development of a shared understanding of a unifying goal-a big-picture question that could only be answered by a collaboration between all our disciplines. However, we did experience some instances where individuals at differing career stages (Figure 1) had to choose which objectives to prioritize over others due to conflicting incentives, as detailed in Sections 3.4.1 and 3.4.2. 


\subsubsection{Misalignment as a Challenge for Data Management: Allocating Time to Tasks}

Although our team members had generally well-aligned goals, many (particularly more senior researchers) struggled to balance time between this project and other responsibilities. We found that sub-teams with a strong graduate student and/or postdoc presence were often the ones that were able to consistently push the project forward. For instance, graduate students and postdocs were frequently the first to respond when a sub-team was asked to share a dataset. In general, we believe that it would be inefficient-or perhaps unrealistic-for leaders of each sub-team to take on the full workload associated with their model or analysis. In addition, some of the early career PIs who were tasked with managing the project in addition to leading scientific tasks felt particularly burdened with trying to accomplish both goals simultaneously. Hiring a project manager mitigated these administrative pressures substantially.

Some of our sub-teams experienced challenges arising from the tradeoffs between model refinement and model coupling. Each model in our project's framework could be extensively refined and calibrated, incorporating the most up-to-date data, the newest software versions, and the latest methodological innovations. In theory, this should lead to increasingly more accurate representations of reality, which is desirable from the perspective of that discipline. However, these refinements take time and may necessitate rerunning other models; every week a sub-team worked to improve its model fit was a week that other sub-teams had to wait before they could couple the models, thus creating a delay in the overall workflow. This problem particularly affects team members with finite funding on the project; for instance, a graduate student may need data from another model to finish their dissertation. For the sake of the larger coupled modeling workflow, we determined that it was often best to compromise, calibrating a model to the extent that it produced outputs that were sufficient for the next model, but we note that knowing how to make this compromise was challenging. Sometimes, a senior co-PI with previous model coupling experience could help to decide the level of sufficiency for a given calibration.

\subsubsection{Misalignment as a Challenge for Co-Authorship: Balancing Authorship Priorities}

While graduate students, postdocs, and pre-tenure faculty share many of the same goals as the rest of our team members, they often face additional pressure to publish papers quickly in disciplinary journals, and to achieve other research outcomes that are important to their career stage. For this reason, we found that these individuals often had the greatest motivation to maintain the project's momentum, especially in terms of publications. This did not generally result in problems, but could cause increased stress when other co-authors did not feel the same pressure and took longer to complete writing tasks.

In some disciplines, this pressure is amplified by career requirements in a team member's field. For example, in the field of economics, the norm for a PhD student to be considered for a tenure-track job is to have a well-polished, discipline-focused paper in which they are the first (or only) author (Cawley 2018). This may, at times, discourage teamwork among these students, because they are forced to prioritize individual, disciplinary papers over collaborative, interdisciplinary publications. In addition, by virtue of the limited authorship criterion for those papers, colleagues may not receive credit for their contributions. This is in contrast to other disciplines, such as ecology and sociology, where research carried out by a single investigator-and thus resulting in a single-author publication-is less common and is potentially viewed as a signal of an inability to work with others (Duffy 2017). These challenges also apply to the choice of journal. Cases in our project arose in which only publications in select disciplinary journals would be valued by the disciplines or institutions of early career researchers, whereas others placed value on journals with a broad readership and high impact.

Based on our experiences, we recommend establishing expectations at the beginning of the project and with the initiation of each manuscript about authorship, turn-around time for writing and revisions, and choice of journal. This can be accomplished with an authorship policy similar to ours (Appendix A). If someone has specific concerns related to authorship, the process of developing and updating this document creates a space where they can air their concerns. During one of the early project workshops for CNH Lakes, our team also set aside time for a candid discussion about each 
member's specific needs based on their career stage. This helped to set expectations, as well as build trust and mutual support among team members.

\subsection{Dimension 5: Permeable Boundaries}

It is normal for team membership to change over the course of a project, with some members leaving and some joining (Gavens et al. 2018). In some cases, this is due to the changing needs of the project-for instance, some types of analysis or modeling may not be needed until the project has been underway for several years, so as to allow enough time for data collection and preliminary analyses. In other cases, it can be a result of the changing lives of the team members-students graduate, postdocs find new positions, and researchers shift their focus. Both of these types of changes drove shifts over time in the composition of our team (Figure 1). These permeable boundaries have the potential to create challenges for effective team functioning by necessitating the development of new interpersonal relationships, as well as the transfer of knowledge and expertise, which both take time and effort.

\subsubsection{Permeable Boundaries as a Challenge for Data Management: Transferring Data}

The CNH Lakes team had several members-primarily postdocs and graduate students-who left the project before it was completed. These individuals made efforts to leave behind well-documented datasets and analyses, so that others would be able to replicate and/or build upon their work. In some cases, this was successful, but in other cases, we found that there were no other team members who had the right combination of time and expertise to continue the work. We also had a number of team members who began working on the project after it was already underway. Given the size and complexity of our project, these individuals had to dedicate a considerable amount of time to familiarizing themselves with the project's research objectives, as well as the tools and datasets that were available for them to use.

For the most part, we found that careful documentation of work and open, honest communication allowed research to continue through team member transitions. However, we also suggest that teams maintain adaptability with regard to the project's specific research objectives, so that if the team gains or loses any given person, it does not derail the goals of the team as a whole.

3.5.2. Permeable Boundaries as a Challenge for Co-Authorship: Incorporating New Team Members as Co-Authors

When individuals joined the team after the project was underway, we often found that it was challenging to determine which manuscripts they should join as co-authors. Manuscript leads had to balance the desire to include new members in relevant team efforts as soon as possible with the knowledge that the magnitude of the project meant that a significant amount had to be learned. There was a risk that joining manuscript development too late would not allow for enough of a contribution to warrant co-authorship.

We believe it is important to have a protocol that guides the process of incorporating new co-authors into manuscript development, as well as maintaining contact with co-authors who leave the project before a manuscript is complete. Having a consistent plan for team members who join and leave the project ensures that important procedures (such as reviewing authorship procedures, or documenting and sharing datasets) are not forgotten. For instance, any individual leaving the team should be given the opportunity to discuss whether or not they will remain involved in any manuscript efforts they had contributed to, and whether their contributions at the time they leave are sufficient for co-authorship. Additionally, if an individual is expected to be a transient member of the team, they should work with the rest of the team to consider the scope of their contributions accordingly, for their benefit as well as for that of the project. 


\subsection{Dimension 6: Geographic Dispersion}

To make use of the best available expertise, resources, and connections, members of interdisciplinary teams often span multiple institutions. Those institutions can range from departments on the same campus to universities on different continents. Team members in different geographic locations may experience challenges arising from communicating exclusively by electronic methods, coordinating across time zones, and meeting different institutional expectations (Hanson et al. 2016; Read et al. 2016; Weathers et al. 2013). Our project had collaborators distributed across more than seven institutions in almost as many states in the U.S., and had to coordinate tasks between geographically dispersed team members from its inception.

\subsubsection{Dispersion as a Challenge for Data Management: Troubleshooting Issues}

Despite the benefits of online tools_including email and videoconferencing-that allow communication between geographically dispersed individuals, there was a consensus among our team members that face-to-face meetings were ideal, which is reinforced by the experience of global research networks (Weathers et al. 2013; Hanson et al. 2016). Throughout the CNH Lakes project, we had numerous experiences in which an in-person meeting resolved confusion and allowed us to push the project forward. These meetings were critical for issues involving highly specialized data transfers, where variable naming conventions were not consistent between disciplines (e.g., meetings between members of the ecologic, agronomic, and hydrologic modeling sub-teams were crucial for understanding the workflow that would be necessary to couple those models). At a similar meeting later on, the team was able to answer questions about a model's calibration by allowing collaborators to discuss, brainstorm, and visualize data in real time. This was because the problem-solving techniques necessary to overcome such difficulties required extensive interaction, real-time troubleshooting, and long meetings. For these reasons, we believe that there are many instances where in-person meetings are the best option available, despite the challenges and costs associated with travel.

\subsubsection{Dispersion as a Challenge for Co-Authorship: Editing Remotely}

A multitude of tools and software are available for co-authors to facilitate collaborative writing. For instance, online tools enable simultaneous editing of a document by multiple parties, and features in word processors can allow the tracking of edits by individual authors. We found that simultaneous editing tends to work best at the early stages of manuscript development, because it allows all co-authors to share their ideas. On the other hand, we found that individual editing using the "track changes" feature and serial editing works best for fine-tuning the manuscript in its later stages, because it keeps the document organized, particularly when there are many co-authors involved.

During the development of the literature review paper discussed in Section 3.2.2, we found that co-authors who worked at the same institution were better able to quickly resolve questions or concerns, because they could more easily hold in-person meetings when necessary. Co-authors at different institutions, however, were often limited to email discussions. When comments were addressed in this manner, the process was generally more time-consuming, and more likely to lead to misunderstandings.

\subsection{Dimension 7: High Task Interdependence}

In interdisciplinary projects, team members depend on each other to complete tasks in order to move the project forward. This requires effective communication, planning, and trust. When delays occur with one component of the project, they are likely to affect multiple other components, potentially leading to delays or conflicts. Managing the interdependence of tasks is a core aspect of running a project with a large team. 


\subsubsection{Interdependence as a Challenge for Data Management: Coupling Models and Analyses}

Model coupling is an inherently interdependent process. Generally, each model must be calibrated, and a model must be fully run in order to produce outputs that may be used as inputs for another model. In CNH Lakes, this interdependence was amplified by the fact that we were coupling not two models, but six. Throughout our project, sub-teams had to carefully coordinate with each other to establish a timeline for when data or outputs would be available to share between models. This was challenging because it was often difficult to accurately predict how long it would take to run or calibrate a model. Additionally, unforeseen problems could arise at various points in the process that required days, weeks, or even months to address.

While waiting to receive input data from other sub-teams, our team members worked to optimize their time by determining what tasks could be accomplished without those data. For example, while waiting for data from the agronomic and hydrological models, the lake modeling team used different, preexisting datasets to run analyses that led to discipline-specific publications. Although these publications did not advance the coupled modeling workflow of our project, they represented a valuable contribution to disciplinary literatures (especially for early career researchers) and pushed the workflows forward so that they were ready when upstream models were completed.

\subsubsection{Interdependence as a Challenge for Co-Authorship: Editing Efficiently}

The same software tools that can facilitate collaboration between large and geographically dispersed sub-teams can also help reduce the inefficiencies caused by high task interdependence. For instance, online document editing software can allow co-authors to work on the sections of a manuscript that are less interdependent, while waiting for other, more integrated sections to be completed.

In our experience, the best strategy for guiding the development of a manuscript (which involves high task interdependence in and of itself) is to combine bottom-up and top-down writing styles. This means allowing all co-authors to have input in the writing process, but also having a specific leader, small leadership team, or advocate who guides writing and pushes the process forward. This ensures that all perspectives are represented, while preventing writing from becoming delayed or from stalling out. The leader does not necessarily need to be the first author; rather, it is simply a person who is designated as having some authority over manuscript development (Frassl et al. 2018; Oliver et al. 2018).

\section{Discussion}

In addressing challenges that arose in our project across the seven dimensions of team science, a recurring theme that emerged is that building the practical day-to-day infrastructure to support effective team communication was essential. It is well-established and accepted within the science of team science and CNHS literatures that frequent, open communication serves myriad purposes, including developing trust, creating a shared conceptual model, translating ideas across disciplinary boundaries, and sharing disciplinary norms and expectations (Alberti et al. 2011; Bennett and Gadlin 2012; Carey et al. 2019; Cheruvelil et al. 2014; Lanier et al. 2018). Few researchers would quarrel with the general assertion that effective communication is critical, yet there is little guidance to be found within the CNHS community on how to implement specific day-to-day practices that support this lofty goal.

Throughout our project, our understanding of how to productively communicate evolved significantly. We initially relied on monthly, one-hour teleconferences to handle project logistics and advance our research objectives. This approach proved ineffective both because time on the calls was too limited to meaningfully address our team science challenges and because engagement in the teleconferences was consistently lackluster, with little back-and-forth conversation between team members. To address the latter issue, we shifted from audio-only teleconferences to a videoconference format and were able to achieve a notable improvement in participation and open dialogue. To 
address the challenge of time limitations, we adopted a three-part strategy that combined monthly videoconferences, more in-depth quarterly check-ins, and annual in-person workshops. Each type and model of communication allowed us to address different team science challenges described in Section 3; taken together, these complementary strategies formed a foundation for improving our team dynamics and research productivity.

Monthly videoconferences formed the core foundation for addressing project administration and cultivating a sense of accountability among researchers. These meetings were particularly helpful in surmounting challenges related to data-sharing conventions (Section 3.1.1), disciplinary norms (Section 3.1.2), versioning control (Section 3.3.1), and allocating time to tasks (Section 3.4.1). The frequency of these meetings and the need to cover logistics meant there was generally insufficient time to discuss research objectives in depth. We introduced quarterly videoconferences, during which each sub-team presented brief slide updates using graphics, images, and data visualizations to document recent accomplishments and share their upcoming objectives. These quarterly check-ins occurred with sufficient frequency to allow us to address challenges due to permeable boundaries, such as transferring data (Section 3.5.1) and introducing new team members as co-authors (Section 3.5.2), as well as with sufficient depth to keep momentum in manuscript development (Section 3.3.2), and edit manuscripts remotely and efficiently (Sections 3.6.2 and 3.7.2). Despite supporting more in-depth discussion of research progress, the quarterly check-ins were no substitute for the depth of knowledge integration possible with in-person interaction. Annual all-hands workshops proved crucial in coordinating analyses across scales (Section 3.2.1) overcoming communication barriers (Section 3.2.2), balancing authorship priorities (Section 3.4.2), troubleshooting issues (Section 3.6.1), and coupling models and analyses (Section 3.7.1).

Our approach to tackling these challenges was necessarily shaped by the specifics of our team and project. Nonetheless, we contend that by sharing the successes and failures of individual projects, the research community can build a body of tailored best practices to support effective teams and advance CNHS science. We often prioritize the details of the research while discussing project management in relatively vague or abstract terms. Bennett and Gadlin (2012) put it succinctly: "Not surprisingly, scientists would rather be doing science than concerning themselves with discussions about how they are all getting along" (p. 7). Yet an attention to the simple logistics of teamwork is necessary to even begin addressing complex questions that sit at the intersection of diverse disciplines. There is a great deal of interest within the science of team science community on sharing case studies about team experiences, but the opportunities to advance this dialogue within the CNHS research community are relatively limited. By sharing our team experience, we hope to advance a necessary, broader dialogue about not just the science of CNHS, but about developing the management infrastructure necessary to support project success.

\section{Conclusions}

Interdisciplinary team science is critical to addressing complex, large-scale socio-environmental issues. Many of the elements that give interdisciplinary teams great strength (diversity, large size, deep integration and interdependence) also pose challenges. Our experience suggests that devoting time upfront to data management planning and co-authorship practices will increase team success. Furthermore, building trust through honest communication and in-person meetings is crucial to reconciling disciplinary norms and balancing authorship priorities. We found that data management and co-authorship are two areas where proactive planning and communication can have the greatest benefits in supporting team collaboration. We encourage other interdisciplinary science teams to share their own experiences to expand upon this growing body of knowledge and foster the success of future interdisciplinary teams. 
Author Contributions: Conceptualization, V.R.H. and K.M.C.; Methodology, V.R.H.; Investigation, V.R.H., K.M.C., K.C.W., C.C.C., K.J.F., J.L.K., M.G.S., N.K.W., and W.W.; Writing - Original Draft, V.R.H. and K.M.C.; Writing Review \& Editing, K.C.W., C.C.C., K.J.F., J.L.K., M.G.S., N.K.W., and W.W.; Visualization, V.R.H.; Supervision, K.M.C.; Project Administration, K.M.C. and C.C.C.; Funding Acquisition, K.M.C., K.C.W., C.C.C., J.L.K., and M.G.S. All authors have read and agreed to the published version of the manuscript.

Funding: This research was funded by the National Science Foundation as part of the Dynamics of Coupled Natural and Human Systems (CNH) Program under award number 1517823.

Conflicts of Interest: The authors declare no conflict of interest. The funder had no role in the design of the study; in the collection, analyses, or interpretation of data; in the writing of the manuscript, or in the decision to publish the results.

\section{Appendix A}

CNH-Lakes Manuscript Authorship Invitation $\mathrm{Memo}^{1}$

TO: All CNH-Lakes team members

FROM: [Fill in lead author(s) names]

MANUSCRIPT TITLE: [Fill in tentative title]

MANUSCRIPT TYPE: [Fill in manuscript type: disciplinary research; graduate student thesis or dissertation; interdisciplinary research; essay, concept, or commentary; data or methods; other (please describe)]

I (We) are contacting you because you have been listed as a potential co-author on the above manuscript that is associated with the CNH-Lakes project. On the next page is a list of potential contributions by co-authors on manuscripts. This list is intended to foster an open dialogue on authorship that starts at the very beginning phase of a manuscript and carries through until manuscript submission and acceptance. This document is intended to clearly define each co-author's responsibilities and accomplishments throughout the effort, as well as the overall strategy for determining co-authorship as described below.

1. If you are interested in being a co-author on this manuscript, we ask that you describe in specific terms the ways that you will contribute to the manuscript. Some examples of potential manuscript contributions are listed on the next page (note that this list is not exhaustive; please contact the lead author(s) if you would like to make contributions not included in the list). For each of these contributions, please be as specific as possible as to your contribution (e.g., instead of "collect data," please specify what data will be collected and how this data collection will occur); this additional step is critical for tracking the progress of this contribution in ODS, as described in the $\mathrm{CNH}$-Lakes Manuscript Authorship Guidelines.

2. Addition of co-authors. We recognize that in some cases it may be impossible to identify all co-authors at the beginning stages of a manuscript. In situations when an individual's expertise is added to a manuscript in the middle of the manuscript development process, they should be added to the author list if their contributions satisfy the conditions described below.

3. This list of potential contributions is not intended to be a checklist: we recognize that there are many different possible types of contributions to manuscripts throughout the initiation, development, analysis, and writing processes and that it is difficult to compare these contributions. Our goal is to be as inclusive and flexible as possible for each person who makes a substantive contribution to the manuscript. Here, we define a substantive contribution as a contribution in which the manuscript would not have been possible without it, or that it substantially enhances the breadth or quality of the manuscript. The specific contributions of each participant will be considered on

1 The effect of cross-scale interactions on freshwater ecosystem state across space and time. PIs: P.A. Soranno, K.S. Cheruvelil, E.H. Stanley, J.A. Downing, N.R. Lottig, P-N. Tan. NSF, Emerging Frontiers Division, Macrosystems Biology Program. 2011-2016. Awards: 1065786, 1065818, 1065649. 
a case-by-case basis and co-authorship status will be determined as the outcome of a discussion between manuscript lead(s), potential co-authors, and if necessary, the CNH-Lakes Steering Team (see the note on conflict resolution below). Some contributions may be more appropriately recognized in the acknowledgments section of a manuscript, rather than with a co-authorship. This determination will be made on a case-by-case basis via discussion between manuscript lead(s) and established co-authors. Exceptions to this guideline: We recognize that all manuscripts may not neatly fit within this guideline. For example:

i. Given the interdisciplinary nature of the $\mathrm{CNH}$-Lakes project, this guideline will need to be flexible to accommodate domain experts who should be listed as co-authors (e.g., helped with conception and model interpretation such that the breadth or quality of the manuscript is enhanced).

ii. Manuscripts that are position-pieces or commentaries may need different criteria.

4. Once contributions are identified, the manuscript lead(s) should create a task for each co-author in ODS. Each co-author should then populate ODS with specific sub-tasks that describe their contributions to the manuscript. We expect the co-authors to regularly update their progress in ODS to ensure transparency with the full $\mathrm{CNH}$ team and enable coordination of manuscript activities within the authorship team.

5. Author ordering will be determined on a case-by-case basis after discussions among all co-authors of the contributions of each co-author throughout the manuscript process. In general, authorship is in order of significance of contributions by each co-author to the final manuscript. However, we recognize that some disciplinary differences exist with respect to authorship position (e.g., the last author indicates lab leadership in some scientific disciplines). It will most often be the case that the manuscript lead(s) will be listed first, followed by co-authors in order of contribution. Where different contributions cannot be compared, an alphabetical listing of co-authors is the recommended practice.

6. In general, data provision is not assumed a priori to warrant co-authorship. In many cases, providing data in and of itself is not considered a contribution significant enough to constitute co-authorship. However, there may be exceptions when significant data processing has been undertaken to make the data usable for this manuscript, the manuscript may not have been possible without the data, or the suggestion of providing the data led to enhancing the breadth or quality of the manuscript. If any data provider expresses an interest in co-authorship, it is the responsibility of the manuscript lead(s) to contact that person to confirm the data provision and other contributions justify co-authorship.

7. All co-authors must approve the final version of the manuscript prior to submission. It is unethical to submit a manuscript in which all co-authors did not read and approve the final submitted version. This task is not included in the contributions list below because all co-authors must do it.

8. Co-authors are held accountable for the content of the manuscript. This idea provides an important distinction between a co-author and someone who is acknowledged. We recognize that every co-author will not have full knowledge of all aspects of the research; however, they need to know enough to defend the work.

9. It is recommended that an author-contribution paragraph be written for each manuscript. This step is important to ensure that all co-authors (particularly early-career team members) are recognized for the contributions that they make to the $\mathrm{CNH}$-Lakes project. Because many journals don't automatically publish these statements, we recommend adding it to the Acknowledgements section in the manuscript.

10. Conflict resolution: As noted above, it is our goal to be as inclusive as possible in the CNH-Lakes project. In the event of a disagreement between contributors and manuscript lead(s) about co-authorship contributions and status, we encourage manuscript lead(s) to err on the side of being inclusive of those who view their contributions as substantive enough to warrant 
co-authorship. In the event of a dispute about authorship or manuscript content, the first stage in conflict resolution is for the lead(s) and the contributor in question to meet with the Steering Team (Cobourn, Carey, and Boyle) to discuss and resolve the disagreement. If the disagreement involves one or more members of the Steering Team, an ad-hoc committee of 3 CNH-Lakes research participants not participating in the manuscript will be formed to review and mediate the dispute.

Examples of Potential Co-author Contributions

Potential co-author contributions identified here are a starting point for $\mathrm{CNH}$ team members to think about whether their contributions to a manuscript rise to the level of co-authorship. This is by no means an exhaustive list of ways in which co-authors may contribute, and not all of these contributions may warrant co-authorship. As the project evolves and different types of manuscripts are created, the contributions made by potential co-authors are likely to vary significantly and should be evaluated on a case-by-case basis.

Examples of Concept and Design Contributions

1. Conceived or contributed to the conception of a manuscript idea/overarching topic such that input helped define the fundamental contribution of the manuscript

2. Developed or fundamentally contributed to formulating research questions

3. Designed/outlined the manuscript

4. Contributed to the conceptual/theoretical framework for the manuscript

5. Supervised and/or co-supervised authors and manuscript progress

6. Provided platform for research to occur (e.g., facilitated interactions with lake associations, created $\mathrm{CNH}-$ Lakes infrastructure that enabled research interactions to occur, etc.)

\section{Examples of Research Contributions}

1. Collected data (e.g., lake association interviews, downloaded data from databases)

2. Compiled or synthesized data (e.g., merged data from different datasets for model activities)

3. Oversaw or led quality assurance/quality control $(\mathrm{QA} / \mathrm{QC})$ of data

4. Developed models or a part of a model

5. Calibrated models

6. Ran or estimated models

7. Integrated models

8. Developed model scenarios

9. Analyzed observed data or model output data

10. Contributed new analyses or methods

11. Interpreted results or placed results in a policy context to enhance the greater contributions of the $\mathrm{CNH}$-Lakes project

Examples of Writing Contributions

1. Wrote sections of text

2. Designed figures and tables

3. Performed critical reviews or substantial re-working of manuscript

Other

We welcome additional contributions and encourage a potential co-author to discuss other contributions with the lead author(s) so that they can be made explicit in ODS. 


\section{References}

Alberti, Marina, Heidi Asbjornsen, Lawrence A. Baker, Nicholas Brozovic, Laurie E. Drinkwater, Scott A. Drzyzga, Claire A. Jantz, José Fragoso, Daniel S. Holland, Timothy (Tim) A. Kohler, and et al. 2011. Research on Coupled Human and Natural Systems (CHANS): Approach, Challenges, and Strategies. Bulletin of the Ecological Society of America 92: 218-28. [CrossRef]

Bennett, L. Michelle, and Howard Gadlin. 2012. Collaboration and Team Science: From Theory to Practice. Journal of Investigative Medicine 60: 768-75. [CrossRef]

Carey, Cayelan C., Nicole K. Ward, Kaitlin J. Farrell, Mary E. Lofton, Arianna I. Krinos, Ryan P. McClure, Kensworth C. Subratie, Renato J. Figueiredo, Jonathan P. Doubek, Paul C. Hanson, and et al. 2019. Enhancing Collaboration between Ecologists and Computer Scientists: Lessons Learned and Recommendations Forward. Ecosphere 10: e02753. [CrossRef]

Cawley, John. 2018. A Guide and Advice for Economists on the U.S. Junior Academic Job Market, 2018-2019 Edition. Available online: https://www.aeaweb.org/content/file?id=869 (accessed on 21 September 2018).

Cheruvelil, Kendra S., Patricia A. Soranno, Kathleen C. Weathers, Paul C. Hanson, Simon J. Goring, Christopher T. Filstrup, and Emily K. Read. 2014. Creating and Maintaining High-Performing Collaborative Research Teams: The Importance of Diversity and Interpersonal Skills. Frontiers in Ecology and the Environment 12: 31-38. [CrossRef]

Cobourn, Kelly M., Cayelan C. Carey, Kevin J. Boyle, Christopher Duffy, Hilary A. Dugan, Kaitlin J. Farrell, Leah Fitchett, Paul C. Hanson, Julia A. Hart, Virginia Reilly Henson, and et al. 2018. From Concept to Practice to Policy: Modeling Coupled Natural and Human Systems in Lake Catchments. Ecosphere 9: e02209. [CrossRef]

Duffy, Meghan A. 2017. Last and Corresponding Authorship Practices in Ecology. Ecology and Evolution 7: 8876-87. [CrossRef]

Eigenbrode, Sanford D., Michael O'rourke, J. D. Wulfhorst, David M. Althoff, Caren S. Goldberg, Kaylani Merrill, Wayde Morse, Max Nielsen-Pincus, Jennifer Stephens, Leigh Winowiecki, and et al. 2007. Employing Philosophical Dialogue in Collaborative Science. BioScience 57: 55-64. [CrossRef]

Frassl, Marieke A., David P. Hamilton, Blaize A. Denfeld, Elvira de Eyto, Stephanie E. Hampton, Philipp S. Keller, Sapna Sharma, Abigail S. L. Lewis, Gesa A. Weyhenmeyer, Catherine M. O’Reilly, and et al. 2018. Ten Simple Rules for Collaboratively Writing a Multi-Authored Paper. Edited by Fran Lewitter. PLOS Computational Biology 14: e1006508. [CrossRef]

Gavens, Lucy, Joanne Holmes, Gerhard Bühringer, Jordache McLeod, Maike Neumann, A. R. Lingford-Hughes, Emma S. Hock, and Petra Sylvia Meier. 2018. Interdisciplinary Working in Public Health Research: A Proposed Good Practice Checklist. Journal of Public Health 40: 175-82. [CrossRef]

Goring, Simon J., Kathleen C. Weathers, Walter K. Dodds, Patricia A. Soranno, Lynn C. Sweet, Kendra S. Cheruvelil, John S. Kominoski, Janine Rüegg, Alexandra M. Thorn, and Ryan M. Utz. 2014. Improving the Culture of Interdisciplinary Collaboration in Ecology by Expanding Measures of Success. Frontiers in Ecology and the Environment 12: 39-47. [CrossRef]

Hanson, Paul C., Kathleen C. Weathers, and Timothy K. Kratz. 2016. Networked Lake Science: How the Global Lake Ecological Observatory Network (GLEON) Works to Understand, Predict, and Communicate Lake Ecosystem Response to Global Change. Inland Waters 6: 543-54. [CrossRef]

Hipsey, Matthew R., Louise C. Bruce, Casper Boon, Brendan Busch, Cayelan C. Carey, David P. Hamilton, Paul C. Hanson, Jordan S. Read, Eduardo de Sousa, Michael Weber, and et al. 2019. A General Lake Model (GLM 3.0) for Linking with High-Frequency Sensor Data from the Global Lake Ecological Observatory Network (GLEON). Geoscientific Model Development 12: 473-523. [CrossRef]

Jones, Natalie A., Sylvie Shaw, Helen Ross, Katherine Witt, and Breanna Pinner. 2016. The Study of Human Values in Understanding and Managing Social-Ecological Systems. Ecology and Society 21: 15. [CrossRef]

Lanier, Alicia L., Jillian R. Drabik, Tanya Heikkila, Jessica Bolson, Michael C. Sukop, David W. Watkins, Jennifer Rehage, Ali Mirchi, Victor Engel, and David Letson. 2018. Facilitating Integration in Interdisciplinary Research: Lessons from a South Florida Water, Sustainability, and Climate Project. Environmental Management 62: 1025-37. [CrossRef]

Lee, You-Na, John P. Walsh, and Jian Wang. 2015. Creativity in Scientific Teams: Unpacking Novelty and Impact. Research Policy 44: 684-97. [CrossRef] 
Liu, Jianguo, Thomas Dietz, Stephen R. Carpenter, Carl Folke, Marina Alberti, Charles L. Redman, Stephen H. Schneider, Elinor Ostrom, Alice N. Pell, Jane Lubchenco, and et al. 2007. Coupled Human and Natural Systems. AMBIO 36: 639-49. [CrossRef]

National Research Council. 2015. Enhancing the Effectiveness of Team Science. Edited by Nancy J. Cooke and Margaret L. Hilton. Washington, DC, USA: National Academies Press.

Oliver, Samantha K., C. Emi Fergus, Nicholas K. Skaff, Tyler Wagner, Pang-Ning Tan, Kendra Spence Cheruvelil, and Patricia A. Soranno. 2018. Strategies for Effective Collaborative Manuscript Development in Interdisciplinary Science Teams. Ecosphere 9: e02206. [CrossRef]

Palmer, Margaret A., Jonathan G. Kramer, James Boyd, and David Hawthorne. 2016. Practices for Facilitating Interdisciplinary Synthetic Research: The National Socio-Environmental Synthesis Center (SESYNC). Current Opinion in Environmental Sustainability 19: 111-22. [CrossRef]

Pascual, Unai, Patricia Balvanera, Sandra Díaz, Gyö rgy Pataki, Eva Roth, Marie Stenseke, Robert T. Watson, Başak Esra Dessane, Mine Islar, Eszter Kelemen, and et al. 2017. Valuing Nature's Contributions to People: The IPBES Approach. Current Opinion in Environmental Sustainability 26-27: 7-16. [CrossRef]

Read, Emily Kara, Michael O’Rourke, Grace S. Hong, Paul C. Hanson, Luke A. Winslow, Stephen J. Crowley, Carol A. Brewer, and Kathleen C. Weathers. 2016. Building the Team for Team Science. Ecosphere 7: e01291. [CrossRef]

Rüegg, Janine, Corinna Gries, Ben Bond-Lamberty, Gabriel J. Bowen, Benjamin S. Felzer, Nancy E. McIntyre, Patricia A. Soranno, Kristin L. Vanderbilt, and Kathleen C. Weathers. 2014. Completing the Data Life Cycle: Using Information Management in Macrosystems Ecology Research. Frontiers in Ecology and the Environment 12: 24-30. [CrossRef]

Schimel, David, and Michael Keller. 2015. Big Questions, Big Science: Meeting the Challenges of Global Ecology. Oecologia 177: 925-34. [CrossRef] [PubMed]

SESYNC. 2012. Socio-Environmental Systems. Available online: https://www.sesync.org/socio-environmentalsystems (accessed on 9 December 2019).

Soranno, Patricia A., Linda C. Bacon, Michael Beauchene, Karen E. Bednar, Edward G. Bissell, Claire K. Boudreau, Marvin G. Boyer, Mary T. Bremigan, Stephen R. Carpenter, Jamie W. Carr, and et al. 2017. LAGOS-NE: A Multi-Scaled Geospatial and Temporal Database of Lake Ecological Context and Water Quality for Thousands of US Lakes. GigaScience 6. [CrossRef]

Stokols, Daniel, Kara L. Hall, Brandie K. Taylor, and Richard P. Moser. 2008. The Science of Team Science Overview of the Field and Introduction to the Supplement. American Journal of Preventive Medicine 35: S77-S89. [CrossRef] [PubMed]

Uriarte, María, Holly A. Ewing, Valerie T. Eviner, and Kathleen C. Weathers. 2007. Constructing a Broader and More Inclusive Value System in Science. BioScience 57: 71-78. [CrossRef]

Van Noorden, Richard. 2015. Interdisciplinary Research by the Numbers. Nature 525: 306-7. [CrossRef]

Ward, Nicole K., Leah Fitchett, Julia A. Hart, Lele Shu, Joeseph Stachelek, Weizhe Weng, Yu Zhang, Hilary Dugan, Amy Hetherington, Kevin Boyle, and et al. 2019. Integrating Fast and Slow Processes Is Essential for Simulating Human-Freshwater Interactions. Ambio 48: 1169-1182. [CrossRef]

Weathers, Kathleen C., Paul C. Hanson, Peter Arzberger, Jennifer Brentrup, Justin Brookes, Cayelan C. Carey, Evelyn Gaiser, David P Hamilton, Grace S Hong, Bas Ibelings, and et al. 2013. The global lake ecological observatory network (GLEON): The evolution of grassroots network science. Limnology and Oceanography Bulletin 22: 71-73. [CrossRef]

Wilkinson, Mark D., Michel Dumontier, IJsbrand Jan Aalbersberg, Gabrielle Appleton, Myles Axton, Arie Baak, Niklas Blomberg, Jan-Willem Boiten, Luiz Santos, Bourne Bonino da Silva, and et al. 2016. Comment: The FAIR Guiding Principles for Scientific Data Management and Stewardship. Scientific Data 3: 160018. [CrossRef]

Wuchty, Stefan, Benjamin F. Jones, and Brian Uzzi. 2007. The Increasing Dominance of Teams in Production of Knowledge. Science 316: 1036-39. [CrossRef]

(C) 2020 by the authors. Licensee MDPI, Basel, Switzerland. This article is an open access article distributed under the terms and conditions of the Creative Commons Attribution (CC BY) license (http://creativecommons.org/licenses/by/4.0/). 